\title{
Analyse Moléculaire De La Diversité Génétique Des Protéines De Réserves Chez Quatre Variétés De Blé Dur (Triticum turgidum L.var durum)
}

\author{
Nabila Adoui, M.A.A \\ Université M'sila, Algérie ; Université Constantine1, Algérie \\ Laid Benderradji, M.C.A. \\ Noura Messaoudi, PhD. \\ Université M'sila, Algérie \\ Faiçal Brini, Prof.
}

Centre de Biotechnologies de Sfax, Université Sfax,Tunisie

Leila Boudour, Prof.

Université Constantine1, Algérie

Doi: 10.19044/esj.2017.v13n36p249 URL:http://dx.doi.org/10.19044/esj.2017.v13n36p249

\section{Abstract}

The molecular polymorphism of the stock proteins, namely, glutenin and gliadin has been studied in order to perform a comparison of the genes coding for these factors in the quality of bread as much as they are major contributors to the elasticity and formation of wheat dough. DNA markers for quality traits are currently being used by wheat breeders to directly measure traits and performing biochemical tests. The aim of this study was to use DNA markers to search for varieties with glutenin genes (HMW and LMW) and gliadin genes also. 4 durum wheat varieties (alexandrium, murcenese, italicum and valencia) were used for these genes using PCR/RFLP markers via (F-56) and (R-56) primers. Results obtained show that polymorphism rate of this two primer is $54.54 \%$. These two primers produce the same number of amplifiers whose size is 536bp. A total of eleven fragments are obtained for each primer. Five are relatively polymorphic, which concerns the genotype: 4, 20, 76, 91 and 92 through the pairing of an expression density materialized by low intensity bands. The study also revealed that there was no product at $527 \mathrm{bp}$, the size of (HMW) glutenin gene; this indicates that the 100 genotypes do not contain this gene. Thus indicating its poverty in this element; compared to the (LMW) subunit of this gene which accounts for about $60 \%$ of glutenin proteins. While there is $770 \mathrm{bp}$ product for the gliadin gene, which indicates also that the 100 genotypes contain this gene. Thus indicating its good quality for bread making. 
Key words: Durum wheat, DNA markers, quality traits, glutenin, gliadin

\section{Résumé}

Le polymorphisme moléculaire des protéines de réserves (glutenine et gliadine), ont été étudiés dans le but d'effectuer une comparaison des gènes codants pour ces facteurs clés dans la qualité du pain d'autant plus qu'ils sont des contributeurs majeurs de l'élasticité et de la formation de la pâte de blé. Les marqueurs d'ADN pour les caractères de qualité sont actuellement utilisés par les sélectionneurs du blé afin de mesurer directement les caractères sans attendre les générations en effectuant ainsi des tests biochimiques. Le but de cette étude réside dans l'utilisation des marqueurs de l'ADN pour la recherche de variétés de blé disposées de gènes de glutenine matérialisé par la sous unité de haut poids moléculaire (HPM) et la sous unité de faible poids moléculaire (FPM) d'une part et de gènes de gliadine d'autre part. Quatre variétés de blé dur (alexandrium, murcenese, italicum et valencia) ont été utilisées pour rechercher ces gènes en utilisant des marqueurs PCR/RFLP et des amorces (F-56) et (R-56). Les résultats obtenus montrent que le taux de polymorphisme des deux amorces (F-56 \& R-56) est de 54,54\%. Les deux amorces ne produisent qu'un même nombre d'amplifiants de taille $(536 \mathrm{pb})$. Au total onze fragments sont obtenus pour chacun des amorces. Sur ces onze fragments ciblés, cinq sont peu polymorphes (génotype: 4, 20, 76, 91 et 92) à travers l'appariation d'une densité d'expression matérialisé par des bandes peu intenses. L'étude a fait sortir aussi qu'il n'y avait pas de produit à $527 \mathrm{pb}$, soit la taille du gène de glutenine (HPM), ceci indique que les 100 lignes ne contiennent ce gène indiquant ainsi sa pauvreté en cet élément clé ; comparativement à la sous unité (FPM) de ce même gène qui représente environ $60 \%$ des protéines de glutenine. Cependant, il y’à de produit à $(770 \mathrm{pb})$ pour le gène de gliadine, ce qui indique que les 100 lignes contiennent ce gène prouvant ainsi sa bonne qualité pour la panification.

Mots clés : Blé dur, marqueur d'ADN, caractères de qualité, glutenine, gliadine

\section{Introduction}

Dans le système agricole, les céréales (blés, orge et riz) occupent une place primordiale à l'échelle mondiale. Elles sont considérées comme une principale source de la nutrition humaine (Slama et al., 2005). Les deux espèces de blé les plus cultivées sont le blé tendre (Triticum aestivum L.) avec plus de $90 \%$ de production mondiale et le blé dur (Triticum durum Desf.) qui constitue 5\% de celle-ci (Gooding, 2009). Selon la FAO (2016), la production mondiale de blé en 2016 s'établirait à 723 millions de tonnes. En 
Algérie, cette production a atteint 3,30 millions de tonnes pendant la compagne agricole 2014/15 (FAO, 2015). Par ailleurs, la filière des céréales constitue la ration alimentaire quotidienne occupant ainsi une superficie de 2.7 millions d'hectares (Benbelkacem, 2013). Étant donné la forte augmentation de la population mondiale dans les années à venir et pour fin de satisfaire la demande alimentaire de l'humanité, il est nécessaire d'augmenter au moins à $60 \%$ la quantité des produits agricoles d'ici jusqu'à 2050 (Gallais, 2015). Pour l'Algérie, les besoins d'une population en pleine croissance, la production est estimée à plus 111 million de quintaux vers 2020 (Hervieu et al., 2006). La production des céréales est devenue une question préoccupante, et cela suppose que le milieu s'y prêt et que la technologie l'encadre. En effet, l'amélioration génétique du blé a eu pendant longtemps pour objectif l'augmentation de la productivité, et le succès de cette stratégie est lié à l'existence de conditions environnementales favorables, qui permettent l'expression des différents facteurs du rendement. Pour se faire, la sélection massale qui est une ancienne méthode de sélection agricole, se base sur le choix des semences et répond le mieux aux critères des paysans (Zeven, 1998). Les céréales sélectionnées pour être cultivées murissaient uniformément en facilitant la moisson, d'autant plus qu'elles se conservaient suffisamment longtemps pour assurer de la nourriture jusqu'à la prochaine moisson et fournissent des semences pour la saison suivante de culture (Marchenay, 1987 ; Yadav et al., 2011). Le but principal de tout programme de sélection est la production de variétés possédant un rendement élevé et stable, dont l'environnement dans lequel se fait la sélection joue un rôle important. Tous les milieux n’ont pas la même aptitude à révéler les différences génotypiques et l'existence d'une interaction (génotype $\mathrm{x}$ environnement), complique les efforts de la sélection (Sanchez-Garcia et al., 2012). Le processus de sélection est différent, selon qu'on cherche l'adaptation spécifique ou l'adaptation générale (Annichiarico et al., 2006). Les marqueurs morphologiques sont déjà connus comme des outils efficaces pour l'estimation de la diversité génétique du blé (Al Khanjari et al., 2008) ainsi que la caractérisation des ressources génétiques est une étape clé pour la sélection (Amallah et al., 2016). De ce fait, les ressources génétiques représentent un patrimoine qu'il est nécessaire de préserver et de mieux exploiter. Ainsi, pour assurer une agriculture durable, l'étude de la diversité et la caractérisation de ces ressources génétiques sont indispensables pour créer de nouvelles variétés ayant une bonne qualité, un rendement élevé, adaptées aux variations climatiques et résistantes aux maladies (Aguiriano et al., 2006). L'objectif de notre étude porte sur l'identification des gènes codants pour les protéines de réserves, à savoir, les glutenines et les gliadines afin de déterminer le polymorphisme et/ou la diversité génétique des variétés étudiées et de mettre en place un système efficace de protection de ces 
variétés afin d'évaluer les caractères de production et d'adaptation pour mieux connaitre ces potentialités génétiques avant de se lancer dans des leurs programmes d'amélioration.

\section{Matériel et méthodes}

Matériel végétal

Le matériel végétal utilisé est composé de 4 variétés de blé dur (Triticum turgidum L.var durum) à savoir, alexandrium (alex), valencia (vale), italicum (ita) et murcenese (mur) regroupant chacune plusieurs génotypes (Tableau 1) (Boudour et al., 2004; Boudour, 2006).

Tableau 1: Caractères des variétés étudiées.

\begin{tabular}{|c|c|c|c|c|c|c|c|}
\hline Caractères & $\begin{array}{c}\text { Couleur de } \\
\text { l'épi }\end{array}$ & $\begin{array}{c}\text { Couleur } \\
\text { de barbe }\end{array}$ & $\begin{array}{c}\text { Couleur } \\
\text { et forme } \\
\text { du grain }\end{array}$ & $\begin{array}{c}\text { Forme de } \\
\text { l'épi }\end{array}$ & Précocité & Compacité & Paille \\
\hline alexandrium & $\begin{array}{c}\text { Glabre } \\
\text { rouge }\end{array}$ & Noire & $\begin{array}{c}\text { Blanc } \\
\text { Allongé } \\
\text { bossu }\end{array}$ & $\begin{array}{c}\text { Fusiforme } \\
\text { à } \\
\text { pyramidal }\end{array}$ & Tardive & Compacte & Creuse \\
\hline valencia & $\begin{array}{c}\text { Pubescent } \\
\text { rouge }\end{array}$ & Blanche & Blanc & Pyramidal & Précoce & $\begin{array}{c}\text { Lâche à } \\
\text { compacte }\end{array}$ & Creuse \\
\hline italicum & $\begin{array}{c}\text { Pubescent } \\
\text { rouge }\end{array}$ & Rouge & Blanc & Pyramidal & $\begin{array}{c}\text { Demi } \\
\text { précoce }\end{array}$ & Compacte & $\begin{array}{c}\text { Demi } \\
\text { creuse }\end{array}$ \\
\hline murcenese & $\begin{array}{c}\text { Glabre } \\
\text { rouge }\end{array}$ & Rouge & $\begin{array}{c}\text { Rouge } \\
\text { Gros } \\
\text { bossu }\end{array}$ & $\begin{array}{l}\text { Fusiforme } \\
\text { Fusiforme }\end{array}$ & Précoce & $\begin{array}{c}\text { Demi } \\
\text { compacte }\end{array}$ & $\begin{array}{c}\text { Demi } \\
\text { pleine }\end{array}$ \\
\hline
\end{tabular}

Le but de cette étude est de faire un screening de différents génotypes pour la présence ou l'absence de gènes de gluten (glutenine et gliadine) de bonne qualité. Il s'agit de 100 génotypes qui ont été regroupées en lots contient chacun 5 génotypes, soit au total 20 lots obtenus (Tableau 2).

Tableau 2: Répartition des génotypes étudiés

\begin{tabular}{|c|c|c|c|}
\hline Lot & Genotypes & Lot & Genotypes \\
\hline $\mathbf{1}$ & alex1, alex2, alex3, alex4, alex5 & 11 & vale 4, vale 5, vale 6, vale 7, vale 8 \\
\hline 2 & alex6, alex8, alex9, mur1, mur2 & 12 & $\begin{array}{l}\text { vale51, vale10, } \text { vale11, } \\
\text { vale13 }\end{array}$ \\
\hline 3 & mur4, mur5, mur6, mur7, mur8 & 13 & $\begin{array}{l}\text { vale14, vale15, } \text { vale16, } \\
\text { vale18 }\end{array}$ \\
\hline 4 & mur9, mur10, mur11, $\mathrm{m}$ & 14 & $\begin{array}{llll}\text { vale19, } & \text { vale20, } & \text { vale21, } & \text { vale22, } \\
\text { vale23 }\end{array}$ \\
\hline 5 & mur14, $\mathrm{m}$ & 15 & $\begin{array}{l}\text { vale25, vale26, } \text { vale27, } \text { vale } 28, \\
\text { vale29 }\end{array}$ \\
\hline 6 & 2, mur23 & 16 & $\begin{array}{l}\text { vale32, vale34, vale35, vale36, } \\
\text { vale37 }\end{array}$ \\
\hline 7 & mur24, mur25, mur26, $\mathrm{n}$ & 17 & $\begin{array}{l}\text { vale38, vale39, vale40, vale43, } \\
\text { vale44 }\end{array}$ \\
\hline 8 & mur $29, \quad$ mur30, mur31, mur32, & 18 & ita 21 , ita22, ita23, ita24, ita25 \\
\hline
\end{tabular}




\begin{tabular}{|l|l|l|l|}
\hline & mur33 & & \\
\hline $\mathbf{9}$ & mur34, mur35, mur36, mur37, mur38 & $\mathbf{1 9}$ & ita26, ita27, ita28, ita29, ita30 \\
\hline $\mathbf{1 0}$ & mur39, mur40, mur41, vale 2, vale3 & $\mathbf{2 0}$ & ita31, ita32, ita38, ita39, ita40 \\
\hline
\end{tabular}

Ces génotypes ont été utilisés pour une amplification PCR/RFLP, ils ont été pris deux à deux, $[(4,7) ;(51,76) ;(88,91) ;(10$ et 11$)]$ pour chaque variété [(alex); (vale); (ital); (mur)] respectivement afin d'effectuer un séquençage d'ADN du gène de gluténine (sous unité : Haut Poids Moléculaire «HPM ») et (sous unité : Faible Poids Moléculaire «FPM ») et du gène de gliadine (Tableau 3 ).

Tableau 3 : Polymorphisme génotypique des variétés étudiées

\begin{tabular}{|c|c|c|c|c|c|c|c|}
\hline $\begin{array}{c}\mathrm{N}^{\circ} \\
\text { génotyp } \\
\mathrm{e}\end{array}$ & $\begin{array}{c}\text { Variété } \\
\text { alexandrium }\end{array}$ & $\begin{array}{c}\mathrm{N}^{\circ} \\
\text { génotype }\end{array}$ & $\begin{array}{c}\text { Variété } \\
\text { valencia }\end{array}$ & $\begin{array}{c}\mathrm{N}^{\circ} \\
\text { génotype }\end{array}$ & $\begin{array}{c}\text { Variété } \\
\text { italicum }\end{array}$ & $\begin{array}{c}\mathrm{N}^{\circ} \\
\text { génotype }\end{array}$ & $\begin{array}{c}\text { Variété } \\
\text { murcenese }\end{array}$ \\
\hline- & alex1 & $\mathbf{5 1}$ & vale4 & $\mathbf{8 8}$ & ital23 & $\mathbf{1 0}$ & mur2 \\
- & alex2 & $\mathbf{7 6}$ & vale32 & $\mathbf{9 1}$ & ital26 & $\mathbf{1 1}$ & mur4 \\
- & alex3 & - & vale48 & $\mathbf{9 2}$ & ital27 & $\mathbf{1 8}$ & mur11 \\
$\mathbf{4}$ & alex4 & - & vale49 & - & ital93 & $\mathbf{2 0}$ & mur13 \\
- & alex5 & - & vale51 & - & ital94 & - & mur36 \\
- & alex6 & - & vale52 & - & ital96 & - & mur40 \\
$\mathbf{7}$ & alex8 & - & vale53 & - & ital97 & - & mur41 \\
- & alex9 & - & vale54 & - & ital98 & - & mur42 \\
& & - & vale67 & - & ital111 & - & mur43 \\
& & - & valen68 & - & ital115 & - & mur44 \\
& & - & vale69 & - & ital118 & - & mur45 \\
& & & & - & ital119 & - & mur46 \\
& & & & & & - & mur47 \\
\hline
\end{tabular}

\section{Caractérisation moléculaire de génotypes étudies}

L'étude moléculaire a porté sur les génotypes sélectionnés et l'extraction de l'ADN génomique de ces génotypes a été réalisée à partir de plantes récoltées des pots de végétation. Seules, les dernières paires de feuilles sont prélevées puis déshydratées par Silica gel sans cobalt pendant une semaine.

\section{Extraction et purification de l'ADN}

Le protocole d'extraction est celui d'un kit commercial, Qiagen DNeasy Plant Mini Kit (Courtaboeuf, France), sans modification. Le protocole de base non commercial est celui de Doyle et al., (1987) à base de détergent cationique CTAB (cetyl trimethylammonium bromide) déjà utilisé par Murray et al., (1980). Le tampon d'extraction étant composé de $2 \%(\mathrm{p} / \mathrm{v})$ de CTAB; 1,4 M NaCl ; 20mM Na 2 EDTA; 100mM Tris-HCl (pH 8) et 1\% de bisulfite de sodium ajouté extemporanément. Environ $20 \mathrm{mg}$ de feuilles déshydratées mises en suspension dans $1 \mathrm{ml}$ de tampon d'extraction préchauffé à $60^{\circ} \mathrm{C}$ sont broyées dans un mortier stérilisé par autoclavage, le 
broyat est incubé à $60^{\circ} \mathrm{C}$ au bain-marie pendant 60 min avec une agitation manuelle et fréquente par inversion des tubes. Après une centrifugation rapide, $500 \mu \mathrm{l}$ d'un mélange phénol/chloroforme/alcool isoamylique 25/24/1 sont ajoutés au surnageant, l'ensemble est agité, centrifugé à $10000 \mathrm{~g}$ pendant $10 \mathrm{~min}$ à $4^{\circ} \mathrm{C}$, puis à la phase aqueuse sont ajoutés $500 \mu \mathrm{l}$ d'un mélange chloroforme/alcool isoamylique (24/1). Après agitation, l'ensemble est centrifugé à $10000 \mathrm{~g}$ pendant $10 \mathrm{~min}$ à $4^{\circ} \mathrm{C}$. A la phase aqueuse, sont ajoutés $2 / 3$ de volume d'isopropanol, le mélange est conservé à $\left(-20^{\circ} \mathrm{C}\right)$ pendant $30 \mathrm{~min}$. Après précipitation, l'ensemble est centrifugé à $10000 \mathrm{~g}$ pendant $10 \mathrm{~min}$ à $4^{\circ} \mathrm{C}$, puis et après élimination du surnageant, le culot est séché dans un évaporateur concentrateur centrifuge sous vide (Speed Vac Savant Instruments, Farmingdale, USA) pendant $5 \mathrm{~min}$ et ré-suspendu en présence de $500 \mu \mathrm{l}$ de $\mathrm{NaCl} 1 \mathrm{M}$ et de RNase à la concentration finale de $800 \mu \mathrm{g} . \mathrm{ml}^{-1}$ pendant $30 \mathrm{~min}$ à $37^{\circ} \mathrm{C}$. Ensuite, $500 \mu \mathrm{l}$ d'un mélange chloroforme/alcool isoamylique $24 / 1$ sont ajoutés. Les tubes agités manuellement et centrifugés à $10000 \mathrm{~g}$ pendant $10 \mathrm{~min}$ à $4^{\circ} \mathrm{C}$ dont à la phase aqueuse sont ajoutés $2 / 3$ de volume d'isopropanol, le mélange est conservé à $\left(-20^{\circ} \mathrm{C}\right)$ pendant $30 \mathrm{~min}$. Après précipitation, l'ensemble est centrifugé à $10000 \mathrm{~g}$ pendant $10 \mathrm{~min}$ à $4^{\circ} \mathrm{C}$, et après élimination du surnageant, le culot d'ADN est séché à l'évaporateur concentrateur centrifuge sous vide pendant $5 \mathrm{~min}$ et résuspendu dans $30 \mu \mathrm{l}$ de TE (10mM Tris (pH 8), 20mM Na 2 EDTA) toute la nuit à $4^{\circ} \mathrm{C}$.

Un témoin d'extraction sans matériel biologique est systématiquement inclue dans la procédure. La quantité et la qualité de l'ADN $(1,5 \mu l)$ sont estimées par dosage au spectrophotomètre Nano-Drop (Thermo Scientific Nano-DropTM8000). L'évaluation de la pureté par DO à $260 \mathrm{~nm}$ et $280 \mathrm{~nm}$ est le rapport DO260 / DO280, et la quantification par DO à $260 \mathrm{~nm}$.

\section{Electrophorèse d'ADN}

L'analyse éléctrophorétique de l'ADN est réalisé avec $3 \mu \mathrm{l}$ d'ADN, ajouté au $2 \mu \mathrm{l}$ de tampon de charge $(5 \mathrm{x})$, à $\mathrm{pH} 8[0.75 \mathrm{ml}$ bleu de bromophénol $(2 \%)$, $5 \mathrm{ml}$ glycérol $(100 \%), 2 \mathrm{ml}$ Na-EDTA, $0.75 \mathrm{ml}$ xylène cyanole $(2 \%)$, $1.5 \mathrm{ml} \mathrm{H} \mathrm{H}_{2} \mathrm{O}$ ]. Le support d'électrophorèse est formé d'un gel d'agarose à $1 \%$ [ $1 \mathrm{~g}$ agarose dans $100 \mathrm{ml}$ de tampon TBE $(1 \mathrm{x})$ à $\mathrm{pH} 8$ en présence $2 \mu \mathrm{l}$ de bromure d'ethilium $(10 \mu \mathrm{g} / \mu \mathrm{l})]$. Après avoir coulé le gel, formation des puits et le laisser polymériser ; les échantillons d'ADN $(5 \mu l)$ qui sont préparés par l'addition de $2 \mu \mathrm{l}$ de tampon de charge au $3 \mu \mathrm{l}$ de l'ADN sont déposés dans les puits et soumis à un courant de 93volts durant 90min, en utilisant le tampon de migration 'TBE $(5 \mathrm{x})(5.4 \mathrm{~g}$ Tris, $0.29 \mathrm{~g}$ EDTA $500 \mathrm{mM}$ à $\mathrm{pH} 8,2.75 \mathrm{~g}$ Acide borique, $100 \mathrm{ml} \mathrm{H}_{2} \mathrm{O}$ ), à $\mathrm{pH}=8$. 


\section{Analyse PCR/RFLP} $770 \mathrm{pb}$

L'analyse RFLP est effectuée par l'utilisation de deux amorces de

F-56:5'GGATCCATGAAGACCTTTCTCATCCT3'

R-56:5'AAGCTTTCAGTTTGTACCGAAGATGC3'

L'amplification de l'ADN à l'aide d'une PCR pour l'analyse RFLP est réalisée dans un thermo-cycler $\mathrm{ADN}$ (Biometra, $\mathrm{GmbH}$ ) selon la méthode de Williams et al., (1990) ; Zietkiewiez et al., (1994). Un volume finale de $25 \mu \mathrm{l}$ contient $2.5 \mu \mathrm{l}$ de tampon PCR $(10 \mathrm{x}), 0.5 \mu \mathrm{l} \mathrm{MgCl}_{2}, 0.5 \mu \mathrm{l}$ dNTP, 0.5 amorce, $2 \mu \mathrm{l}$ ADN, 0.5 Unité Taq-polymérase et $16 \mu \mathrm{H}_{2} \mathrm{O}$ (Tableau 4). Cette amplification comprend une phase de dénaturation initiale d'ADN de $2,5 \mathrm{~min}$ à $94^{\circ} \mathrm{C}$, une phase de 30 cycles avec $1 \mathrm{~min}$ à $94^{\circ} \mathrm{C}$, , pour la dénaturation de $1^{\prime} \mathrm{ADN}, 1 \mathrm{~min}$ à $37^{\circ} \mathrm{C}$ pour l'hybridation des amorces, $1 \mathrm{~min}$ à $72^{\circ} \mathrm{C}$ pour l'élongation et une phase d'élongation finale de $10 \mathrm{~min}$ à $72^{\circ} \mathrm{C}$. On y trouve d'abord une phase de dénaturation initiale de $2 \min$ à $94^{\circ} \mathrm{C}$, suivie de 40 cycles pour une dénaturation de $94^{\circ} \mathrm{C}$ pendant 30 secondes, pour une hybridation à $52^{\circ} \mathrm{C}$ pendant 45 secondes et pour une extension à $72^{\circ} \mathrm{C}$ pendant 2 min et enfin un dernier cycle d'extension finale à $72^{\circ} \mathrm{C}$ pendant 7 min. Après $2 \mathrm{~h}$, Les bandes d'ADN amplifiés sont visualisées sous lumière ultraviolette avec $10 \mu \mathrm{g} / \mu \mathrm{l}$ de bromure d'éthilium et la taille des fragments est estimée sur la base d'une échelle d'ADN de 100 à 2000 paires de base.

Tableau 4: Composantes du volume final de la PCR

\begin{tabular}{|c|c|}
\hline Tampon de réaction 10X (50mM KCl, $10 \mathrm{mM}$ Tris- $\mathrm{HCl}, 1.5 \mathrm{mM} \mathrm{MgCl} 2, \mathrm{pH} 9.0)$ & $2.5 \mu \mathrm{l}$ \\
\hline $50 \mathrm{mM} \mathrm{MgCl} 2$ & $0.5 \mu \mathrm{l}$ \\
\hline $2 \mathrm{mM}$ dNTP & $2.5 \mu \mathrm{l}$ \\
\hline $10 \mathrm{mM}$ amorce & $0.5 \mu \mathrm{l}$ \\
\hline $50 \mathrm{ng}$ ADN & $2.0 \mu \mathrm{l}$ \\
\hline 0.5Unité Taq-polymérase & $1.0 \mu \mathrm{l}$ \\
\hline $\mathrm{H}_{2} \mathrm{O}$ & $16 \mu \mathrm{l}$ \\
\hline Volume finale & $\mathbf{2 5 \mu l}$ \\
\hline
\end{tabular}

\section{Analyse de gènes de gluten}

\section{Gènes de glutenine (HPM et FPM)}

Les100 génotypes ont été groupés dans des tubes de 5 génotypes chacun, soit 20 tubes au total, les amorces ( 1 et 2) \& (3 et 4) Ont été utilisée pour amplifier le gène de gluténine (HPM) et (FPM), respectivement

F-HPM: 5'GAAACCTGCTGCGGACAAG3'........1

R-HPM: 5'GCCTAGCAACCTTCACAATC3'.......2

F-FPM: 5'AAACAGAATTATTAAAGCCGG3',.....3

R-FPM: 5'GGTTGTTGTTGTTGCAGCA3'.........4 


\section{Gène de gliadine}

L'amorce suivante a été utilisée pour amplifier le fragment d'AND de taille de 770bp. F-56: 5'GGATCCATGAAGACCTTTCTCATCCT3' R-56: 5'AAGCTTTCAGTTRGTACCGAAGATGCC3'

\section{Analyse phylogénétique et d'expression des gènes}

Les gels sont photographiés, scannés, puis analysés par le logiciel Bio-Rad. Model Gel Documentation 2000. Pour les gels PCR/RFLP, les bandes de chaque profil sont désignées par leur présence $(+)$ ou absence $(-)$. La matrice ainsi établie pour le marqueur généré par les deux amorces est analysée à l'aide du logiciel Bio-Rad. Les résultats obtenus sont traités par l'analyse statistique multi variée. La matrice de l'identité génétique et la distance sont estimés selon le coefficient de Nei (Nei, 1978), en utilisant le logiciel Genetix version 4.05 (Belkhir et al., 2004).

L'analyse phylogénétique a été menée dans MEGA6 (Tamura et al., 2013), à l'exception des séquences répétitives, L'arbre phylogénétique a été construit en utilisant la méthode UPGMA dont le niveau d'expression des gènes a été mesuré par qRT-PCR (Bio-Rad). L'expression génétique a été normalisée au niveau d'expression du gène de la sous unité $15 \mathrm{kDa}$ de l'ARN polymérase (Stephenson et al., 2007).

\section{Résultats et discussion}

\section{Analyse RFLP}

La qualité de 1'ADN a été vérifiée par le rapport DO260/DO280, sa valeur oscille entre 1.92 et 1.95 ce qui montre que le degré de pureté est élevé. L'étude de la pureté de l'ADN avait pour objectif de mettre en évidence le polymorphisme moléculaire des génotypes de blé dur sélectionnés en utilisant le marqueur PCR/RFLP. Le marqueur RFLP est dispersé dans les régions non codantes du génome et plus susceptible de muter en comparaison avec les gènes codants, c'est un indicateur de variations génétiques dans les études phylogénétiques. L’homogénéité de la densité des bandes reflète le nombre presque identique de copies pour la séquence amplifiée. Ce résultat corrobore avec celui de (Noma et al., 2016) qui trouvent chez le blé tendre des copies multiples estimé de (10-15) à (3540) et que le nombre de fragments ne diffère pas par rapport à l'amorce utilisée. De ce fait l'amorce que nous avons utilisée a répondu négativement en nombres de bandes et s'est avéré non polymorphe et que chaque bande correspond à l'amplification de la même séquence d'ADN amplifiée. Le taux de polymorphisme des deux amorces, à savoir, (F-56) \& (R-56) est de 44\%, et cela reflète aussi le taux polymorphique de chaque amorce séparément (Tableau 5, Figure 1). 


\section{Caractérisation des fragments des amorces (F-56 ; R-56)}

Les deux amorces produisent le même nombre d'amplifiants de taille $536 \mathrm{pb}$. Au total 11 fragments sont obtenus à raison d'un fragment pour chaque amorce (F-56; R-56). De ce fait on a distingué pour l'échantillon7 (génotype, alex8); l'échantillon10 (génotype, mur2); l'échantillon11 (génotype, mur4); l'échantillon18 (génotype, mur11); l'échantillon51 (génotype, vale4) et l'échantillon88 (génotype, ital23) qu'il ya une présence de bandes intenses de 536pb. Alors que pour l'échantillon4 (génotype, alex4); l'échantillon20 (génotype, mur13); l'échantillon76 (génotype, vale32); l'échantillon91 (génotype, ital26) et l'échantillon92 (génotype, ital27), on a distingué la présence d'un nombre de bandes moins intenses (Tableau 6, Figure 1).

Tableau 5: Analyse RFLP (génotypes générés par amorces F-56 et R-56).

\begin{tabular}{|c|c|c|c|c|c|c|c|c|c|c|c|c|c|c|}
\hline \multirow[t]{2}{*}{ RFLP } & \multirow{2}{*}{$\begin{array}{c}\mathrm{P} \\
(\%)\end{array}$} & \multirow[t]{2}{*}{$\mathrm{M}$} & \multirow[t]{2}{*}{$\mathrm{PM}(\mathrm{pb})$} & \multicolumn{11}{|c|}{ Echantillon } \\
\hline & & & & 4 & 7 & 10 & 11 & 18 & 20 & $\overline{51}$ & 76 & 88 & 91 & 92 \\
\hline \multirow{13}{*}{$\begin{array}{c}\mathrm{F}-56 \\
\& \\
\mathrm{R}-56\end{array}$} & \multirow{13}{*}{54,54} & 1 & 1130 & - & - & - & - & - & - & - & - & - & - & \\
\hline & & 2 & 1070 & - & - & - & - & - & - & - & - & - & - & - \\
\hline & & 3 & 961 & - & - & - & - & - & - & - & - & - & - & - \\
\hline & & 4 & 865 & - & - & - & - & - & - & - & - & - & - & - \\
\hline & & 5 & 806 & - & - & - & - & - & - & - & - & - & - & - \\
\hline & & 6 & 708 & - & - & - & - & - & - & - & - & - & - & - \\
\hline & & 7 & 610 & - & - & - & - & - & - & - & - & - & - & - \\
\hline & & 8 & 536 & + & + & + & + & + & + & + & + & + & + & + \\
\hline & & 9 & 477 & - & - & - & - & - & - & - & - & - & - & - \\
\hline & & 10 & 418 & - & - & - & - & - & - & - & - & - & - & - \\
\hline & & 11 & 339 & - & - & - & - & - & & - & - & - & - & - \\
\hline & & 12 & 260 & - & - & - & - & - & - & - & - & - & - & - \\
\hline & & 13 & 181 & - & - & - & - & - & - & - & - & - & - & - \\
\hline & \multicolumn{3}{|c|}{ Total $=11$} & 1 & 1 & 1 & 1 & 1 & 1 & 1 & 1 & 1 & 1 & 1 \\
\hline
\end{tabular}

M : marqueur moléculaire, $(4,20,76,91,92)$ : Echantillons des génotypes (alex4, mur13, vale32, ital26, ital27)...bandes moins intenses ; (7, 10, 11, 18, 51, 88): Echantillons des génotypes (alex 8, mur2, mur4, mur11, vale4, ital23).....bandes intenses. $(+)$ : présence, $\quad(-)$ : absence

Tableau 6 : Echantillons des génotypes sélectionnés pour le séquençage de l’ADN.

\begin{tabular}{|c|c|c|c|}
\hline Echantillon & Genotype & Echantillon & Genotype \\
\hline 4 & alex4 & 51 & vale4 \\
\hline 7 & alex8 & 76 & vale32 \\
\hline 10 & mur2 & 88 & ita23 \\
\hline 11 & mur4 & 91 & ita26 \\
\hline 18 & mur11 & 92 & ita27 \\
\hline 20 & mur13 & $/$ & $/$ \\
\hline
\end{tabular}




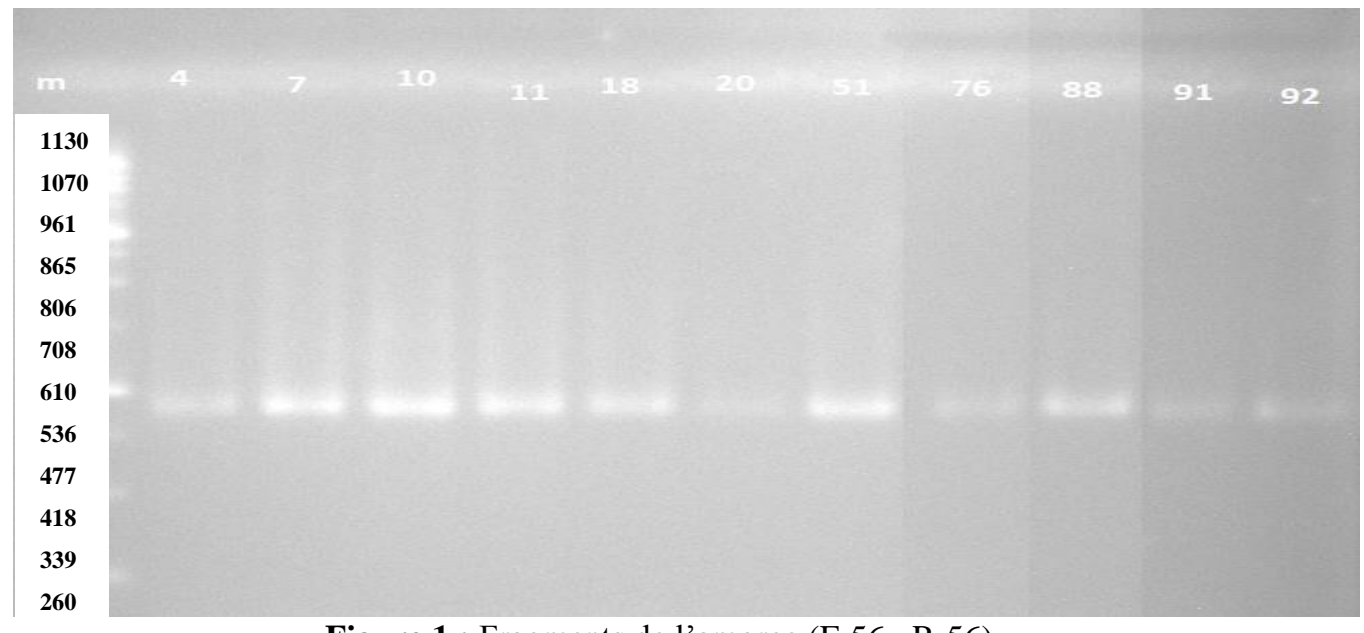

Figure 1 : Fragments de l'amorce (F-56; R-56)

\section{Gène de glutenine (HPM)}

Le gel obtenu des tubes numérotés de 1 à 20 de 100 génotypes, montre de très faibles bandes à $527 \mathrm{pb}$ ce qui confirme l'absence de gènes de glutenine (HPM) et cela indique que ces génotypes dépourvues de ce gène (Figure 2). Selon Abdel-Mawgood (2008) ; Noma et al., (2016), l'absence du gène de gluténine (HPM) a été confirmée par la comparaison avec un témoin de l'ADN du blé tendre égyptien qui a montré des bandes très visible

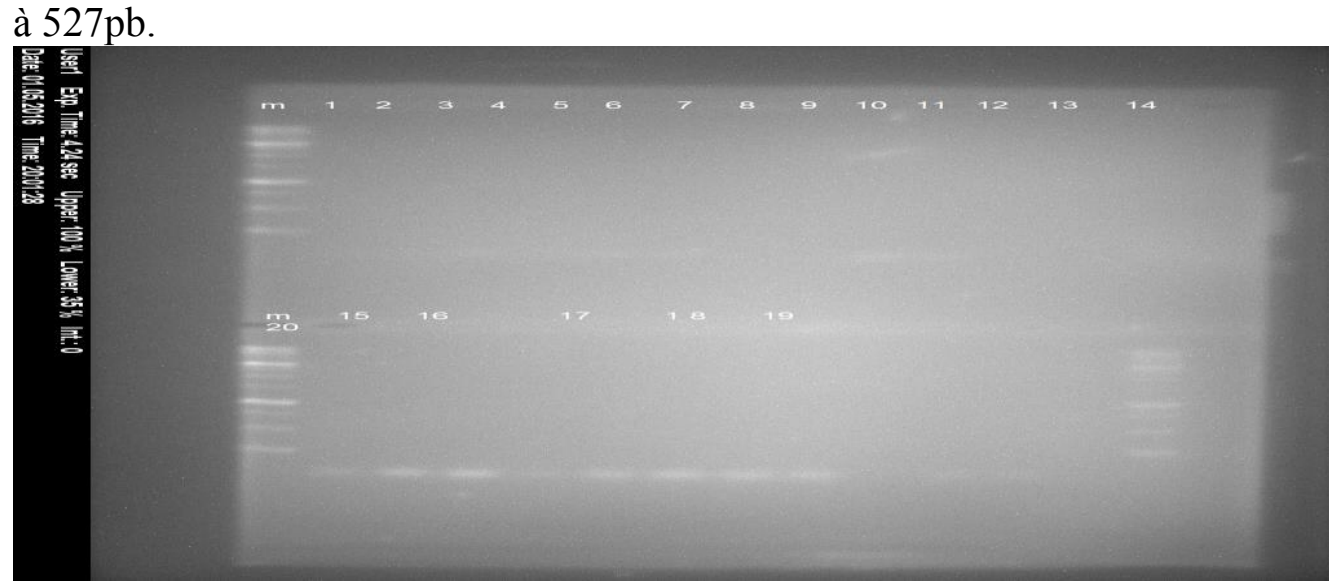

Figure 2 : Absence de bandes du gène de glutenine (HPM)

\section{Gène de glutenine (FPM)}

Le gène de gluténine (FPM) représente environ $60 \%$ des protéines de glutenine dans les graines matures et joue un rôle important dans la formation du macro-polymère de glutenine et de la qualité du gluten, en particulier pour l'extensibilité et la résistance de la pâte. La sous unité (FPM) du gène de glutenine appartient à la famille de gènes multiples. Une étude 
récente basée sur le screening de la bibliothèque $\mathrm{BAC}$ et l'analyse protéomique a montré que Glu-A3, Glu-B3 et Glu-D3 dans le cultivar de blé chinois Xiaoyan54 contiennent 4,3 et 7 gènes respectivement (AbdelMawgood, 2008).

\section{Gène de gliadine}

Le gel obtenu dans les vingt tubes de 100 génotypes étudiés, fait apparaitre une forte présence de bandes correspondent au gène de gliadine, c'est le produit à $770 \mathrm{pb}$ (taille du gène de gliadine). Ceci indique que les 100 génotypes pourvus de ce gène (Tableau 2, Figure 3). Globalement, l'abondance élevée de gène de gliadine est très marqué dans les tubes $1,2,5$, $6,7,8,9,11,12$ comparativement à la faible abondance dans les tubes 3,4 , $10,13,19$, alors que les tubes $14,15,16,17,18$ sont plus abondants en ce gène et plus particulièrement dans le tube numéro 20.

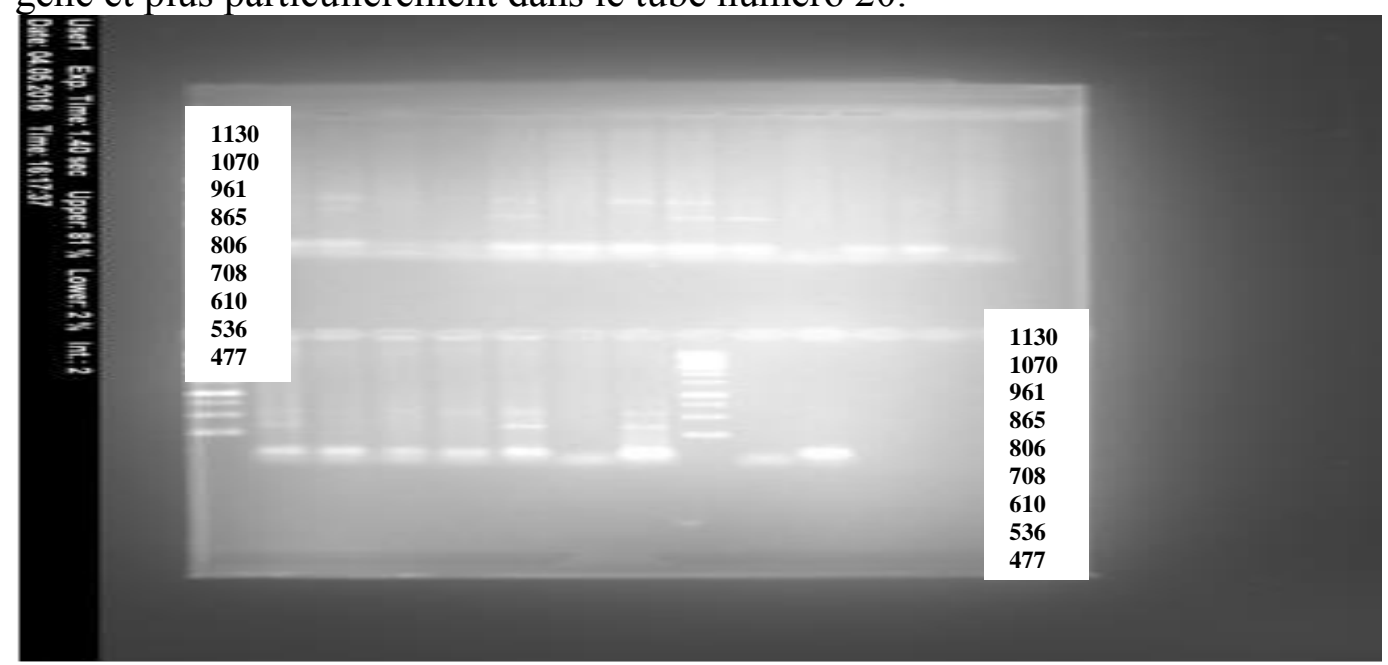

Figure 3 : Gène de gliadine

\section{Analyse phylogénétique et d'expression des gènes}

$\mathrm{Au}$ total, 99 séquences de gène de gliadine avec leurs régions promotrices sont obtenues. Après élimination de 30 séquences redondantes, on aura 69 séquences dont 7 sont de peudogènes avec un codon stop prématuré et 62 étaient intacts avec une séquence de codage complète.

L'analyse phylogénétique a été effectuée en utilisant ces séquences, et les gènes de gliadine ont été classés en onze groupes et nommés selon les noms des variétés (Kawaura et al., 2005). La répartition de ces gènes en fonction des lots de génotypes étudiés (Tableau. 2), est la suivante: Le lot 4 et 7 appartient à la variété alexandrium, le lot 10,11, 18, 20 concerne la variété murcenese, alors que le lot 51 et 76 appartient à la variété valencia et finalement le lot 88, 91, 92 qui sont pour la variété italicum (Figure 4). 
ALEX4

MUR2

ITA25

VOL 4 TA

VOL 32

ITA23

ALEX4

MUR2

MUR2

I TA25

VOL 4

VOL 32
ITA23

ALEX4

ALEX8

MUR2

ITA25

VOL 4

VOL 32

ALEX 4

ALEX

MUR2

MUR4

ITA25

VOL 4

HTA2

ALEX4

ALEX8

MUR2

MUR4

ITA25

VOL 4

VOL 32

ITA23

ALEX 4

ALEX8

MUR2

ITA25

ITA 25
VOL 4

VOL 4

ITL 23

ALEX4

ALEX8

MUR2

MUR4

ITA25

VOL 4

VOL 32
ITA23

ALEX4

ALEX8

MUR2

MUR4

ITA2 25

VOL 4

VOL 32
ITA2

ALEX 4

ALEX8

MUR2

MUR4

ITA 25

VOL 4

VOL 32

ITA23

TGTCTACATGGTAATGAGTAAGATCAGTCTAAACCAC-... TC- - СAATCTACACTAG .... GCGGGGGAGGAGTCCTGC-AATCG--TGGAG_..... AC- - CCAACCG-CCCAC . . GCGAA... ACATATATGATATACACA .... AGTT TATATCCCCCC.. CCCGGAACACTAGAT -

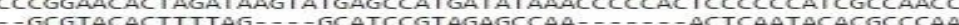
-GCGTACACT T TTAG - - GCATCCGTAGAGCCAA -..... ACTCAATACACGCCCA

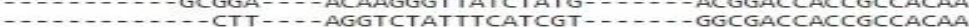

CCAGAGAACT TGCTCTCCTATGA--CTACCGGCGA-- -AACT TAT TACTCATATCTTGTA GCGGGCTTAATGTCCCCGTCTCA--CTTGCAGCCA---AACT TAT TTTCGAAGCGCTGTA - CGCCTCCTC-TTTCCCCCCGCCCATTTTCCCCCTCCCCACCCCCTTTTCAACAAAAC-CACTGCCTTGTAGCTCCCCGCCCTTTTGCCCCCCCCAAATCCCTCTCTCAAACAACCAC TTCCCTTTCCTGATGCCCTCCCC-.TGTGCACTCCCATTTCCGCACTATCGCAC-....CTGCATTTAGAGTTCCAGTOCCACAATTOCAGC-CCAAAATCCATCTCTTCAACAGCCAC CTGCAGTTAGAGTTCCAGTGCCACAATTGCAGCCACAAAATCCATCTCAGCAACAGCCAC CTGCAGTTAGAGTTCCAGTGCCACAATTGCAGCCACAAAATCCATCTCAGCAACAGCCAC

TACTGCCTTTTCT AAGGCGATAACTACTGGT-AACAACTATCTATTACCA CСGAATAATT TCT-...... TATACTGT TT T TGCTGGT-AATAACTATGAGTATAAC CACAGCCATCCCCATTATTTTCCAGCCCTGT TTTTTCTTTTCTCTCCCCTACCCTCTCTC CTCACCAAGCCTCATATCCTAGCCATCCGCGTTGTCTTTTTATCTTCCCCATATCT-DTACCAGCAAGTCCCATTGGTCCCACAACCACAAT TTCTTGGGTTGCTACAACCTTTTCTAC ACCAGCAAGTCCCAT GGT CCCACALCCACAAT AAGAGCAAGT TCCAT TGGTACAACAACAACAATTTCTAGGGCAGCAACAACCATTTCCAC

CGCTTTATCTCAAACTCAAGTGTATGACCTTCTAACATCG-TACGTCATGTT-TCCCCTA AGCTTTATTTGAAACTGAAGTGTATGATCCTTTAACACATGTATGGCATGTTTCACCATA TCTCTTTT--ATTTT TT TT TCCTATGCCTTT TC-TTTCTTTCTCT TCAT TTT TT TT TTT CCCTTITT--CTTTTTCTGGTGTATGATTCTTT-TTTCTTITTCTTCATTTTGTTCTTT - CCTTTCC--CAATTTCGCCCGCCTTCCCCCTT TCCGCCCCGCTTCATATTCTTCTGC CACAACAA- CCATATCCGCAGCCGCAMCCATTTCCATCACAACAACCATATCTGCAGCT CACAACAA--CCATATCCACAGCCGCAACCATT TCCATCACAACAACCATATCTGCAGCT

ATTTAATTATCCCTAAAAT ...............AATAATTGTGTCTGCTACGTCACATTC ATT TAAATATACCTAAAAG ...............AA-TAATTGTGGTGTTACGTCAAATGG CTTCCCTTTTTCCCAGCCA-n. GTTCCCTTTTCCCCAGCCC ..............AACTTCTTTTT-TTTTTTCCTCCCTTTT - TTTCTTCTCCCCAGGAGTACTACCTCCGTACCCTTCTTTCCTCTCCTCCCGCTTCTTC

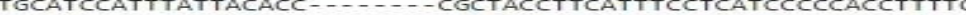

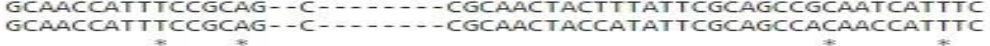

CCTAATTTATTTTATTGGGGGACATGCTCGCTCTTCTATTATCCCTAACCTCAACCCTTC CAGAATTTATTGTAT TGGGGGATATGATGGCTCTTCCATTATACCGAACGTAAAACCTTA TATTATTTGTTTTATACC ............ ATTTTTATTATTCTCCTCСTCTCCTTT-. I - ITTTTGTTTATTATTATCATCTTCTCTA ThT CACCACAAC GACCACAACAACCATATC .......... CACAACCGCAACCACAGTATTCGCAACC..

CGATCACCCCACGAACGCATGCCCCCCACGGGCCACCTTCCATACCCCCCCC-TCTCCAT TGATCACCGCATGAAAGCATGGGTAGAGGTGGCACCTTCCAATACCCCCACA-TCATCAT TGATCACCGCATGAAAGCATGGGTAGAGGTGGCACCTTCCAATACCCCCACA-TCATCAT CCCTCTCCTATT TT-.. TTTTTCTTCAGAAAAGACAATCCCCCTCAACCTCCTTTTGTGC TAGTCTCTCTCCTTTCCCTTTTCAACAACCTTCCAGGTCACACCCCAACACT-TCTTCCC TCT CACCCTCCCC-...TTTCCACCCCCCCCCCACTTCTCCCCCCAC- -TC-TCCTGAT ACAACAACCCCTTI... TTCAGCAGCAGCAGCAGCATCAACAACAACAACA-ACAACAA ACAACAACCAAT TT -... CACAGCAGCAGCAGCAACAACAACAACAACAACA-ACAACAA

CTATGTCCGTTGCCTT- - -TCTCCCCACATTTTCTACTCCTACCCCCCTCCTTCT-TCTC CTATGTGTGTTGCTTT - - - TGATAATAAAAT TTATGTCACTGGTAGAACTAATGT - TTAG CTCTTGCTCTTT TTT TT TT TCCTCCCTTCTT TT TT TCACT TAACATAAT TTATTATTACA CCCTT-CAGCTGTTTCTTTCCCTTCCTTCTTTTCTTCGAACAAAAAAAAAAAAAATAG-AGCA CTCTTACT TCTTTGTT-.ACAAT TAAATTCCCCT TGAATCT TACGCAGTTTTAA-CAGC CCCTTTCTTCATTCCC- TCATTAAATTCCCCTTTCCCATTCCTACATAAGATT-TGTT CCCCTACTACTACTAC-TCCACAATAATACCCTTGCTCCACCTCCAACAGTAA-TACC CAACAACAACAACAAC - - AACAACAACTACAACT TCAACTACAGCAACACT TAT-TGTA

A--TTATCTCTTTTTTTATCATCTTACGCAAGTTACTACCAATTAATACATTTTATCATA AGATTACCTTCGATT TGAGTATCACACGAAGAATAGAACCAACGAGAACCGTTTCCCCAT GAAGTTACA-.G--TGTTTTTGTTAGCGGGGTTTTCTTTC

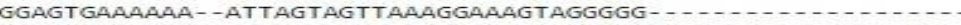

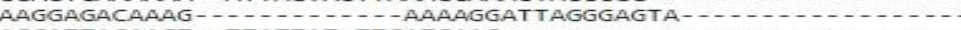
AGCATTACAAGT - - TTATTAT-TTGATGAAG

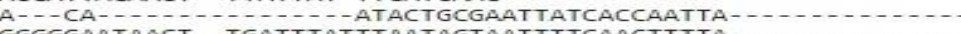
GGGGGAATAACT - TGATTTATTTAATAGTAATTTTCAACTTTTA 


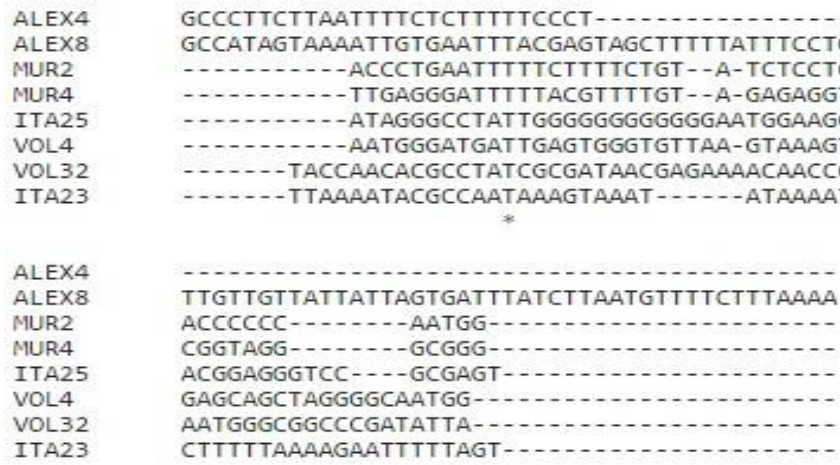

Figure 4. Analyse d'expression des gènes

Caractérisation de fragments de gènes de glutenine et gliadine : Abondance en guanine et cytosine (GC)

La caractérisation par amorce de fragments de gènes de glutenine est non significative à cause de l'absence totale de la sous unité (HPM) de ce gène, ainsi la faible abondance de la sous unité (FPM) de ce même gène, mais elle est significative pour le gène de gliadine (Tableau 7, figure 5). En effet, pour la variété alexandrium, le lot 4 contient $41.37 \%$ de GC (alex4) et 69.04\% (alex8); le lot 7 contient $44.44 \%$ de GC (alex4) et $48.27 \%$ de GC (allex 8). Alors que pour la variété murcenese, le lot 10 contient $48.33 \%$ de GC (mur2) et $47.45 \%$ de GC (mur4), alors que pour le lot 11 , il contient $21.05 \%$ de GC (mur2) et $26.31 \%$ de GC (mur4); le lot 18 contient $45.83 \%$ de GC (mur2) et $40.42 \%$ de GC (mur4) et le lot 20 contient $24.44 \%$ de GC (mur2) et $20.93 \%$ de GC (mur4). Quand à la variété italicum, le lot 51 contient $38.59 \%$ de GC (ita23) et $49.15 \%$ de GC (ita25), le lot 76 contient $33.92 \%$ de GC (ita23) et $33.92 \%$ de GC (ita25) également. Cependant pour la variété valencia, le lot 88 contient $25 \%$ de GC (val4) et $30.76 \%$ de GC (val32), le lot 91 contient $33.33 \%$ de GC (val4) et $40 \%$ de GC (val32) alors que le lot 92 contient $63.15 \%$ de GC (val4) et $52.62 \%$ de GC (val32) (Tableau. 7).

Tableau 7. Taux de (GC \%) de différents génotypes étudiés

\begin{tabular}{|c|c|c|c|c|c|c|c|c|c|c|c|c|c|c|c|}
\hline Var & lot & Gén & GC\% & var & lot & Gén & GC\% & var & lot & Gén & GC\% & var & lot & Gén & GC\% \\
\hline \multirow{7}{*}{ alex } & \multirow{6}{*}{4} & \multirow{3}{*}{$\begin{array}{c}\text { alex } \\
4\end{array}$} & \multirow[t]{3}{*}{41.37} & \multirow{7}{*}{ mur } & \multirow{3}{*}{10} & mur & 48.33 & \multirow{7}{*}{ ita } & \multirow[t]{3}{*}{51} & ita23 & 38.59 & \multirow{7}{*}{ vale } & \multirow[t]{3}{*}{88} & val4 & 25 \\
\hline & & & & & & mur & 47.45 & & & ita25 & 4915 & & & val32 & 3076 \\
\hline & & & & & & 4 & & & & & & & & & \\
\hline & & \multirow{3}{*}{$\begin{array}{c}\text { alex } \\
8\end{array}$} & \multirow[t]{3}{*}{69.04} & & \multirow{3}{*}{11} & mur & 21.05 & & \multirow[t]{3}{*}{76} & ita23 & 33.92 & & \multirow[t]{3}{*}{91} & val4 & 33.33 \\
\hline & & & & & & 2 & & & & & & & & & \\
\hline & & & & & & $\begin{array}{c}\text { mur } \\
4\end{array}$ & 26.31 & & & ita25 & 33.92 & & & val32 & 40 \\
\hline & 7 & $\begin{array}{c}\text { alex } \\
4\end{array}$ & 44.44 & & 18 & $\begin{array}{c}\text { mur } \\
2\end{array}$ & 45.83 & & & & & & 92 & val4 & 63.15 \\
\hline
\end{tabular}




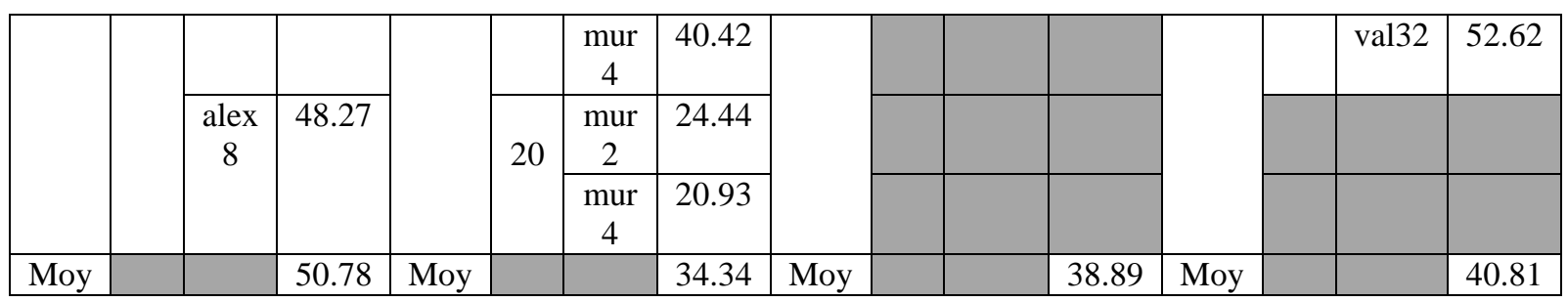

Var = variété, Gén = génotype, GC \% = pourcentage de Guanine et de Cytosine, alex = alexandrium, mur $=$ murcenèse, ita $=$ italicum, val $=$ valencia, Moy $=$ Moyenne

Similarité de Séquences de gène de gliadine

Les différentes variétés étudiées sont classés en 3 groupes sur la base de l'analyse de séquences de gène de gliadine matérialisé par le taux de (GC) et la matrice de l'identité et la distance génétique. Selon l'arbre phylogénétique établi (Figure 6), le premier groupe comporte la variété alexandrium (alex), alors que le deuxième groupe englobe la variété murcenese (mur) tandis que le troisième groupe renferme la variété valencia (vale). Il est à signaler que la variété italicum $(38.89, \mathrm{GC})$ se positionne entre la variété valencia $(40.81 \%, \mathrm{GC})$ et la variété murcenese $(34.34 \%, \mathrm{GC})$ (Figure 5).
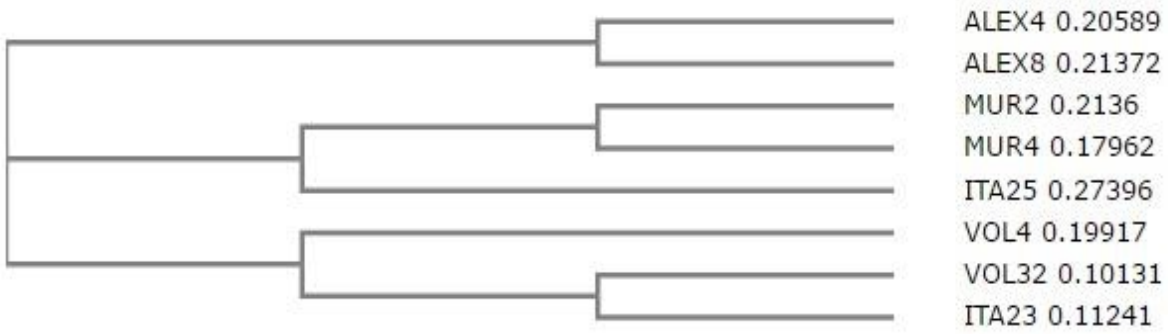

Figure 5 : Analyse phylogénétique des variétés étudiées.

\section{Conclusion}

A travers cette étude, il en résulte que la variété alexandrium (alex) dispose d'une forte stabilité génétique matérialisé par un taux plus élevé de (GC), allant jusqu'à $50.78 \%$, ce qui lui confère une plus grande stabilité de son génome et par conséquent, elle ne va pas aboutir à avoir une diversité et/ou polymorphisme de protéines de réserve et plus particulièrement le gliadine. Cependant, la variété murcenese (mur) s'est avéré la plus divergente avec un taux de (GC) autour de 34.34\%. Alors que la variété valencia (vale) et italicum (ita) sont moyennement stable du point de vue génétique avec un taux de (GC) atteignant $40.81 \%$ et $38.89 \%$ respectivement et par conséquent, elles ménagent une diversité ou un polymorphisme dans leur protéines de réserves et plus particulièrement la gliadine. 
Les résultats obtenus montrent que la diversité génétique chez le blé dur (Triticum turgidum L. var durum) peut être déterminée par l'utilisation des marqueurs moléculaires pouvant ainsi amener à une variation entre les variétés étudiées, et par conséquent l'information retenue pourrait être utile pour les sélectionneurs de génotypes de blé pour augmenter la variation génétique. En outre, l'utilisation efficace des ressources génétiques dans tous les programmes de sélection et amélioration des plantes nécessite auparavant des connaissances approfondies concernant la diversité génétique par le biais d'autres types de marquage moléculaire.

\section{References:}

1. Aguiriano E., Ruiz M., Fité R., Carrillo J. M. (2006). Analysis of genetic variability in a sample of the durum wheat (Triticum durum Desf.) Spanish collection based on gliadin markers. Gen Res and Crop Evolution, 53(8): 1543-1552.

2. Annichiarico P., Bellah F., Chiari T. (2006). Repeatable genotype x location interaction and its exploitation by conventional GIS-based cultivar recommendation for durum wheat in Algeria. Europ. J.Agronomy., 24:70 - 81.

3. Ahmed Abdel-Mawgood, L. (2008). Molecular markers for predicting end-products quality of wheat (Triticum aestivum L.). African journal of biotechnology 7 (14), pp 2324-2327.

4. Al Khanjari S., Filatenko A. A., Hammer K., Buerkert A. (2008). Morphological spike diversity of Omani wheat. Gen Res and Crop Evolution, 55(8), 1185-1195.

5. Amallah L., Hassikou R., Rhrib K., Gaboun, F., Ennadir J., Bouazza F., Taghouti, M. (2016). Analyse de la diversité génétique d'une collection de blé dur par les marqueurs agro-morphologiques et biochimiques. J. Mater. Env.Sci.,7(7):2435-2444.

6. Belkhir, K., Borsa, P., Goudet, J., Chikhi, L. \& Bonhomme, F. (2004). GENETIX, V.4.05 logiciel sous Windows pour la génétique des variétés. Laboratoire de génome, variétés et interactions CNRS UMR 5000, Univ Montpellier II, Montpellier.

7. Boudour, L., Djekoun, A., Ghribi, D., Olmi A. (2004). Morphology diversity of Algerian durum wheat variété. Arab.Univ. J. Sci, 12(2), 597- 608 .

8. Boudour, L. (2006). Étude des ressources phytogénétiques du blé dur (Triticum durum Desf.) Algérien : Analyse de la diversité génétique et des critères d'adaptation au milieu. Thèse Doctorat d'Etat. Université Mentouri Constantine, 142p.

9. Benbelkacem, A. (2013). Rapport national des activités du projet : INRA-ICARDA, 2012/2013: 45p. 
10. Doyle J. J., Doyle J. L. (1987). A rapid DNA isolation procedure for small quantities of fresh leaf tissue. Phytochem Bull 19:11-15

11. F. A. O. (2015). Organisation des nations unies pour l'alimentation et l'agriculture: Perspectives de récolte et situation alimentaire 2015. In, FAO, La carte FAO, 16p. http:/www. fao.org/giews/english/listserv.htm (consultée mars 2015).

12. F. A. O. (2016). Organisation des nations unies pour l'alimentation et l'agriculture: Perspectives de récolte et situation alimentaire 2016. In, FAO, La carte FAO, 6p. http://www. fao. org (consulter Mars 2016).

13. Gooding, M. J. (2009). The wheat crop. In: Wheat chemistry and technology, Khan, K., Shewry P. R, eds. St. Paul, MN: $A A C C$ International, 19-38.

14. Gallais, A. (2015). Comprendre l'amélioration des plantes. Enjeux, méthodes, objectifs et critères de sélection. Eds Quae, 240p.

15. Hervieu, B., Capone, R., Abis, S. (2006). The challenge posed by the cereals sector in the Mediterranean. Ciheam analytical note, (9): 14 p.

16. Kawaura, K., Mochida, K., Ogihara, Y. (2005). Expression profile of two storage-protein gene families in hexaploid wheat revealed by large-scale analysis of expressed sequence tags. Plant Physiol 139:1870-1880.

17. Noma, S., Kawaura, K., Hayakawa, K., Abe, C., Tsuge, N., Ogihara, Y. (2016). Comprehensive molecular characterization of the $\alpha / \beta$-gliadin multigenes family in hexaploid wheat. Mol. Genet. Genomics 291: 65-77.

18. Slama A., Ben Salem M., Zid E. (2005). Les céréales en Tunisie: Production, effet de la sécheresse et mécanismes de résistance. Science et changements planétaires / Sécheresse, 16(3) :225-229.

19. Stephenson, T. J., McIntyre, C. L., Collet, C., Xue, G. P. (2007). Genome-wide identification and expression analysis of the NF-Y family of transcription factors in Triticum aestivum. Plant Mol. Biol. 65: 77-92.

20. Sanchez-Garcia, M., Álvaro, F., Martín-Sánchez, J. A., Sillero, J. C., Escribano, J., Royo C. (2012). Breeding effects on the genotype $\mathrm{x}$ environment interaction for yield of bread wheat grown in Spain during the $20^{\text {th }}$ Century. Field Crops Research, 126:79-86.

21. Tamura, K., Nei, M., Peterson, D., Filipski A., Kumar S. (2013). MEGA6: Molecular evolutionary genetics analysis. Mol. Biol. Evol. 30: 2725-2729.

22. Marchenay, P. (1987). À la recherche des variétés locales de plantes cultivées : Guide méthodologique. Paris, Page Paca, 125 p. 
23. Nei, M., 1978. Estimation of average heterozygosity and genetic distance from a small number of individuals. Genetics, 89, 583-590.

24. Williams, J. J. G. K., Kubelik, A. R., Livak, K. J., Rafalski, J. A., Tingey, S. V. (1990). DNA polymorphism amplified arbitrary primers are useful as genetic markers. Nucl. Acids Res. 18, 65316535

25. Yadav, S. S., Redden, R. J., Hatflield, J. L., Lotze-Campen, H., Hall, A. E. (2011). Crop adaptation to climate change, eds. Oxford, Blackwell, $595 \mathrm{p}$.

26. Zietkiewiez, E., Rafalski, A., Labuda, D. (1994). Genome fingerprinting by simple sequence repeat (SSR) anchored polymerase chain reaction amplification. Genomics, 20(2): 176-183.

27. Zeven, A. C. (1998). Landraces: A review of definitions and classifications. Euphytica 104(2): 127-139. 